\title{
Mosaic analysis of insulin receptor function
}

\author{
Tadahiro Kitamura, ${ }^{1}$ Yukari Kitamura, ${ }^{1}$ Jun Nakae, ${ }^{1}$ Antonio Giordano, ${ }^{2}$ Saverio Cinti, ${ }^{2}$ \\ C. Ronald Kahn, ${ }^{3}$ Argiris Efstratiadis, ${ }^{4}$ and Domenico Accili ${ }^{1}$ \\ ${ }^{1}$ Department of Medicine, College of Physicians \& Surgeons of Columbia University, New York, New York, USA \\ ${ }^{2}$ Department of Anatomy, University of Marche, Ancona, Italy \\ ${ }^{3}$ Joslin Diabetes Center, Harvard University, Boston, Massachusetts, USA \\ ${ }^{4}$ Department of Genetics, College of Physicians \& Surgeons of Columbia University, New York, New York, USA
}

Insulin promotes both metabolism and growth. However, it is unclear whether insulin-dependent growth is merely a result of its metabolic actions. Targeted ablation of insulin receptor (Insr) has not clarified this issue, because of early postnatal lethality. To examine this question, we generated mice with variable cellular mosaicism for null Insr alleles. Insr ablation in approximately $80 \%$ of cells caused extreme growth retardation, lipoatrophy, and hypoglycemia, a clinical constellation that resembles the human syndrome of leprechaunism. Insr ablation in $98 \%$ of cells, while resulting in similar growth retardation and lipoatrophy, caused diabetes without $\beta$-cell hyperplasia. The growth retardation was associated with a greater than 60 -fold increase in the expression of hepatic insulin-like growth factor binding protein-1. These findings indicate that insulin regulates growth independently of metabolism and that the number of insulin receptors is an important determinant of the specificity of insulin action.

J. Clin. Invest. 113:209-219 (2004). doi:10.1172/JCI200417810.

\section{Introduction}

Insulin regulates fuel utilization and storage, as well as cellular proliferation (1). Despite considerable progress in understanding insulin signaling, it is unclear what engenders the specificity of the biologic response. Among the outstanding questions is whether insulin promotes growth independently of metabolism, or whether the two actions are interdependent (2). We have previously demonstrated that constitutive ablation of the insulin receptor (Insr) results in a mild defect in embryonic growth, attributable to decreased Igf2 signaling $(3,4)$. In contrast, ablations of $\operatorname{Ig} f 1$ and its receptor Igflr result in marked dwarfism at birth (5). These data support a model in which growth and metabolism result from distinct cellular pathways, the

Received for publication January 9, 2003, and accepted in revised form November 3, 2003

Address correspondence to: Domenico Accili, Russ Berrie Medical Science Research Pavilion, 1150 St. Nicholas Avenue, Room 238A, New York, New York 10032, USA.

Phone: (212) 851-5332; Fax: (212) 851-5331;

E-mail: da230@columbia.edu.

Conflict of interest: The authors have declared that no conflict of interest exists.

Nonstandard abbreviations used: insulin receptor (Insr); forkhead transcription factor 01 (Foxo1); insulin-like growth factor binding protein-1 (Igfbp1); phosphatidylinositol-tris-phosphate (PIP3); pancreatic and duodenal homeobox protein-1 (Pdx1); peroxisome proliferative activated receptor, $\gamma$, coactivator $1 \alpha$ (Pgc1 $\alpha$ ); heat shock promoter (HSP); HSP 70-1 Cre transgenic (Hs-Cre6); growth hormone (GH); triglyceride (TG); ${ }^{125}$ I-labeled insulin ( ${ }^{125}$ I-insulin); ${ }^{125}$ I-labeled IGF2 ( ${ }^{125}$ I-IGF2); brown adipose tissue (BAT); phosphoenol-pyruvate carboxykinase (Pck1); glucose-6phosphatase (G6pc); hepatic glucose transporter Glut2 (Slc2a2); glucokinase (Gck); sterol regulatory element binding transcription factor-1 (Srebf1); glycogen synthase (Gys1); glycogen phosphorylase (Pygl); phosphofructokinase, liver (Pfkl); forkhead transcription factor a2 (Foxa2); uncoupling protein-1 (Ucp1); peroxisome proliferator activator receptor $\gamma($ Ppar $\gamma)$; adrenergic $\beta_{3}$ receptor (Adrb3). former being primarily regulated by Igf signaling and the latter by insulin signaling (6). Moreover, conditional mutagenesis of the Insr is generally devoid of effects on growth, suggesting that insulin does not have growth-promoting effects independent of its metabolic effects (7).

A second, related question is whether, within the same cell type, different insulin responses display different sensitivities to activation of insulin signaling. The issue has profound practical implications because, although defects of insulin action (i.e., insulin resistance) predispose to type 2 diabetes, many of the complications of insulin resistance (e.g., obesity, hypertension, and infertility) appear to arise from excessive rather than decreased insulin signaling. Because insulin acts through a single receptor, the most widely held view is that specific effects arise from signaling elements at a post-receptor level. Extensive tissue-specific mutagenesis of Insr has begun to address these questions, but there are inherent limitations to this approach, since the pattern and extent of tissue recombination is dependent on the specificity of the promoter used to drive Cre expression, which is rarely - if ever - limited to a single tissue or cell type and, even within a given cell type, is generally limited to a specific developmental stage (8). Moreover, it has become apparent that homeostatic mechanisms enable mice to compensate for impaired insulin signaling in a given tissue by shifting substrate utilization to other tissues (9). To circumvent these limitations, we have exploited the powerful genetics of the Cre-loxP system to generate mice with variable degrees of cellular mosaicism for null Insr alleles (10).

\section{Methods}

Reagents. We purchased anti-Insr (C-19) and anti-IGF1 receptor (C-20) antibodies from Santa Cruz Biotech- 
nology Inc. (Santa Cruz, California, USA), anti-insulin and anti-glucagon antisera from Linco Research Inc. (St. Charles, Missouri, USA), anti-forkhead transcription factor 01 (anti-Foxo1) and anti-phosphoS253 Foxo1 from Cell Signaling Technology Inc. (Beverly, Massachusetts, USA), anti-dynamin, anti-insulin-like growth factor binding protein-1 (anti-Igfbp1), anti-Igfbp2, and antiIgfbp4 from Upstate Biotechnology Inc. (Lake Placid, New York, USA), anti-phosphatidylinositol-tris-phosphate (anti-PIP3) from Echelon Research Laboratories Inc. (Salt Lake City, Utah, USA). We obtained the anti-pancreatic and duodenal homeobox gene-1 (antiPdx1) antiserum from C. Wright (Vanderbilt University, Nashville, Tennessee, USA) and the anti-peroxisome proliferative activated receptor, $\gamma$, coactivator $1 \alpha$ (antiPgc1 $\alpha$ ) antiserum from B. Spiegelman (Harvard University, Cambridge, Massachusetts, USA).

Animal production and phenotypic analysis. The Columbia University Institutional Animal Care and Utilization Committee have approved all animal procedures (Protocol no. 2715-3). We have described previously the generation of Insfrflox (11) mice, in which exon 4 of Insr is flanked by loxP sites, and heat shock promoter (HSP 70-1) Cre transgenic ( $\mathrm{Hs}$-Cre6) mice (10). To generate Insr mosaics, we intercrossed Insrlox/lox and $\mathrm{Hs}$-Cre6 mice to obtain Insrlox/lox, Hs-Cre hemizygous mice. These were then backcrossed with Insrlox/lox mice to obtain mosaics. Thus, in the ensuing progeny, the $\mathrm{Hs}$-Cre transgene was always hemizygous. In the initial set of experiments, we genotyped the resulting progeny by RT-PCR of tail RNA, carried out as described below and exemplified in Figure 1, $\mathrm{a}$ and $\mathrm{b}$. However, in subsequent experiments, we assigned individual mice to either the $\Delta 80$ or $\Delta 98$ categories post hoc, based on the levels of Insr protein detected on a liver Western blot performed after sacrificing the animals. We carried out metabolic analyses as described previously (12), and measured immunoreactive growth hormone (GH) (Linco Research Inc.) and IGF1 (Diagnostic Systems Laboratories Inc., Webster, Texas, USA) using RIA kits. We performed growth analysis as described (13). We measured tissue glycogen content as described previously (14). We measured triglycerides (TGs) with the Trig GB kit (Roche Diagnostics, Basel, Switzerland) and FFA with the NEFA C Test kit (Wako Chemicals USA Inc., Richmond, Virginia, USA).

Western blotting, insulin binding, and ligand blot assays. For Western blotting, we homogenized tissues $(50 \mathrm{mg})$ in buffer containing $20 \mathrm{mM}$ Tris (pH 7.5), $10 \mathrm{mM}$ EGTA, $10 \mathrm{mM} \mathrm{MgCl}_{2}, 1 \% \mathrm{NP}-40,1 \mathrm{mM}$ PMSF, $1 \mathrm{mM}$ DTT. We clarified the lysate by centrifugation at $10,000 \mathrm{~g}$ for 30 minutes at $4{ }^{\circ} \mathrm{C}$, and resolved proteins on SDS-PAGE.

We carried out ${ }^{125} \mathrm{I}$-labeled insulin ( ${ }^{125} \mathrm{I}$-insulin) binding as described (15). We expressed binding as a percentage of WT values, after subtraction of nonspecific binding. We used tissues from Insr $\mathrm{KO}$ mice as controls. For ${ }^{125}$ I-labeled IGF2 (125I-IGF2) ligand blot assays, we used the protocol described by Ooi et al. (16) and used $5 \mathrm{ml}$ of serum. In some experiments, serum was immunoprecipitated with anti-Igfbp 1 , anti-Igfbp2, or
anti-Igfbp4 antisera prior to gel electrophoresis and transfer to nylon membranes.

Histologic analysis. We removed brown adipose tissue (BAT), livers, and pancreata from WT, $\Delta 80$, and $\Delta 98$ mice, fixed and embedded them in paraffin, and mounted consecutive 5 - to $10-\mu \mathrm{m}$ sections on slides. For adipose tissue and liver, we stained sections with $H \& E$. We immunostained pancreata for $\beta$ and $\alpha$ cells using anti-insulin and anti-glucagon antibodies, respectively. For immunofluorescence with anti-Pdx1 and anti-Foxo1 antibodies, we prepared frozen pancreas sections. We used anti-Pdx1 and anti-Foxo1 antibodies at dilutions of 1:5,000 and 1:30, respectively. We used FITC-conjugated secondary anti-rabbit IgG to visualize the immune complexes.

Light microscopy and EM. We fixed small tissue fragments in $2 \%$ glutaraldehyde and $2 \%$ paraformaldehyde in $0.1 \mathrm{M}$ phosphate buffer ( $\mathrm{pH} 7.4$ ), postfixed in $1 \%$ $\mathrm{OsO}_{4}$, dehydrated them in ethanol, and embedded them in an Epon-Araldite mixture (Epon: Multilab Supplies, Fetcham, United Kingdom; Araldite: Fluka Chemie, Buchs, Switzerland). Semithin sections $(2 \mu \mathrm{m})$ were stained with toluidine blue $\mathrm{O}$; thin sections were obtained with a MT-X ultratome (RMC, Tucson, Arizona, USA), stained with lead citrate, and examined with a Philips CM10 transmission electron microscope (Royal Philips Electronics, Eindhoven, The Netherlands).

PIP3 immunohistochemistry. Mice were anesthetized with pentobarbital, and insulin $(1 \mathrm{mU})$ or normal saline was administered via the portal vein, followed 3 minutes later by perfusion with $4 \%$ paraformaldehyde in $0.1 \mathrm{M}$ phosphate buffer ( $\mathrm{pH}$ 7.4). Liver samples were removed and fixed overnight in $4 \%$ paraformaldehyde, followed by $25 \%$ sucrose overnight at $4^{\circ} \mathrm{C}$. They were snap-frozen in OCT Compound (Sakura Finetechnical Co., Tokyo, Japan) and sectioned using a cryostat to yield $7-\mu \mathrm{m}$-thick sections. Liver sections were equilibrated in PBS at room temperature after blocking with 5\% goat serum and $2 \%$ bovine serum in PBS, and were then incubated with mouse anti-PIP3 antibody (Echelon) at a 1:100 dilution overnight at $4^{\circ} \mathrm{C}$. The antibody was detected with cyanin-3-conjugated goat anti-mouse antibody at a 1:200 dilution (Jackson Immunoresearch Laboratories Inc., West Grove, Pennsylvania, USA). After washing with PBS, the sections were counterstained with DAPI (Molecular Probes Inc., Eugene, Oregon, USA) to identify the nucleus.

Immunohistochemical and morphometric analyses. Pancreata were removed from 3-week-old mice, weighed, and fixed overnight in $4 \%$ paraformaldehyde. Sections ( $4 \mu \mathrm{m}$ thick) were immunostained for $\beta$ cells using mouse anti-insulin antibodies and for $\alpha$ cells using mouse anti-glucagon antibodies (Sigma-Aldrich, St. Louis, Missouri, USA). For morphometric analysis of $\beta$-cell mass, three animals of each genotype were analyzed. For each pancreas, three sections spaced at least $40 \mu \mathrm{m}(\Delta 80$ and $\Delta 98$ mice) and $160 \mu \mathrm{m}$ apart (WT mice) were covered systematically by accumulating images from nonoverlapping fields that were captured 
with a digital camera (Nikon 950; Nikon Inc., Melville, New York, USA) and analyzed using the NIH Image 1.60 software, as described (17). Results were expressed as a percentage of the total surveyed pancreatic area occupied by $\alpha$ and $\beta$ cells. $\beta$-cell mass was calculated by multiplying $\beta$-cell area by pancreatic weight.

Real-time RT-PCR and Northern blot analysis. We isolated mRNA from liver tissue and BAT using the MicroFast Track 2.0 kit (Invitrogen Corp., San Diego, California, USA). We performed Northern blot analysis according to standard methods. For semiquantitative RT-PCR analysis to evaluate the efficiency for excision of the "floxed" Insr, we used a set of primers located in Insr exon 3 (5'-CTGTTCGGAACCTGATGA-3') and exon 5 ( $5^{\prime}$-GTGATACCAGAGGATAGGAG- $\left.{ }^{\prime}\right)$. We performed realtime RT-PCR using primer sets encoding Igfbp1, phosphoenol-pyruvate carboxykinase (Pck1), glucose-6-phosphatase (G6pc), hepatic glucose transporter Glut2 (Slc2a2), glucokinase (Gck), sterol regulatory element binding transcription factor 1 (Srebf1), glycogen synthase (Gys1), glycogen phosphorylase $(P y g l)$, phosphofructokinase, liver (Pfkl), Foxo1, forkhead transcription factor-a2 (Foxa2), uncoupling protein-1 (Ucp1), peroxisome proliferator activator receptor $\gamma(P p a r \gamma), P g c 1 \alpha$, and adrenergic $\beta_{3}$ receptor (Adrb3). Primer sequences are available upon request. For these experiments, we isolated mRNA from more than eight mice for each genotype and amplified mRNA from each mouse individually using a Roche Light Cycler PCR instrument and Light Cycler RT-PCR kit (Roche Perkin-Elmer, Foster City, California, USA). We carried out each reaction in triplicate, using a standard curve with the relevant cDNA for each primer set.

\section{Results}

Generation of Insr mosaics. To generate mice with variable degrees of Insr inactivation, we used a binary system based on Cre-loxP recombination. We have previously shown that mice expressing the Cre recombinase under transcriptional control of the HSP 70-1 promoter
(Hs-Cre6) can be used for mosaic analysis of mice carrying floxed alleles (10). In $\mathrm{Hs}$-Cre6 mice, a transient activation of Cre expression occurs in a stochastic manner in the two-cell embryo (10), yielding progeny with cellular mosaicism for gene deletions, when these transgenics are crossed with responder floxed mice. In the offspring, every tissue will be composed of an admixture of cells carrying either intact or null alleles of the gene of interest. Because Cre acts catalytically (18), the likelihood of obtaining heterozygous cells carrying a combination of intact and deleted alleles is, for all intents and purposes, negligible. We have shown that the degree of mosaicism varies between $0 \%$ and $100 \%$ (10).

We crossed mice bearing a floxed Insr allele (11) with $H s$-Cre 6 transgenics (10). The resulting Insr ${ }^{l o x} /+H s$-Cre 6 mice were backcrossed with Ins ${ }^{\text {lox }} /$ lox mice to obtain Ins ${ }^{l o x} /$ lox $\mathrm{Hs}$-Cre 6 mice. These mice displayed different degrees of cellular mosaicism for null alleles of Insr, ranging between less than 5\% and greater than $98 \%$. We measured the degree of Insr inactivation by an RT-PCR assay on liver mRNA that amplified both a 500-bp product derived from the WT Insr allele and a 350-bp product, derived from the deleted allele (Figure 1a). We confirmed the extent of depletion of the protein product by immunoblotting with anti-Insr antiserum (Figure 1b). As a control, IGF1 receptor (Igf1r) levels were unchanged (Figure 1b). To increase the frequency of the desired Ins $r^{b x} / 0 x \mathrm{Hs}$-Cre6 phenotype, we intercrossed Insv ${ }^{l o x} / \mathrm{lox} \mathrm{Hs}$-Cre 6 mice with a low degree of mosaicism (less than 5\%) with Insr $r^{l o x} / 10 x$ mice to generate mosaics carrying the transgene in the hemizygous state. Among offspring from these crosses, we selected two subsets of mosaic mice for further analysis: the first subset had an approximately $80 \%$ reduction of Insr levels (thereafter referred to as $\Delta 80$ ) (Figure 1a, lane 2). A second subset had an approximately 98\% reduction of Insr levels (thereafter referred to as $\Delta 98$ ) (Figure 1a, lane 5). The reduction of Insr protein levels in different tissues within each subset of mice was similar, with brain tissue a
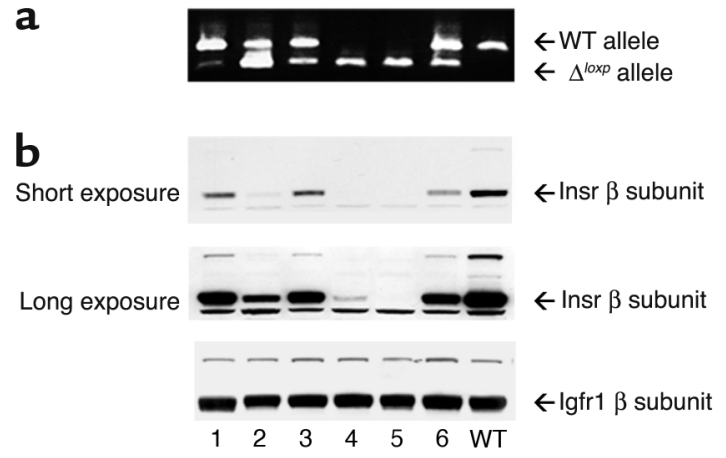

C

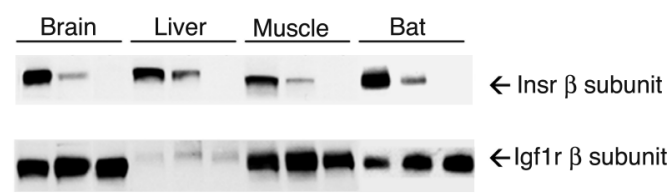

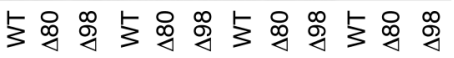

\begin{abstract}
Figure 1
Evaluation of Insr mosaicism. (a) RT-PCR analysis. We isolated mRNA from liver of individual mosaic (lanes 1-6) and WT (lane 7) mice. Because of the deletion of Insr exon 4, the length of the PCR product generated from the $\Delta^{\operatorname{loxP}}$ allele is smaller (350 bp) than the WT allele (500 bp) (upper panel). (b) Protein levels of Insr and Igf1r were examined by Western blotting as indicated in Methods. The first and second panels from the top show different exposures of the same autoradiogram to better visualize Insr expression in mice with greater degrees of mosaicism. The third panel from the top shows samples from the same set of mice analyzed with anti-Igf1r antiserum to normalize protein levels. (c) Expression level of Insr in various tissues in $\Delta 80$ and $\Delta 98$ mice by Western blotting. We removed brain, liver, skeletal muscle, and BAT and determined protein levels of Insr and Igf1 $\mathrm{r}$ by Western blotting as indicated in Methods.
\end{abstract}



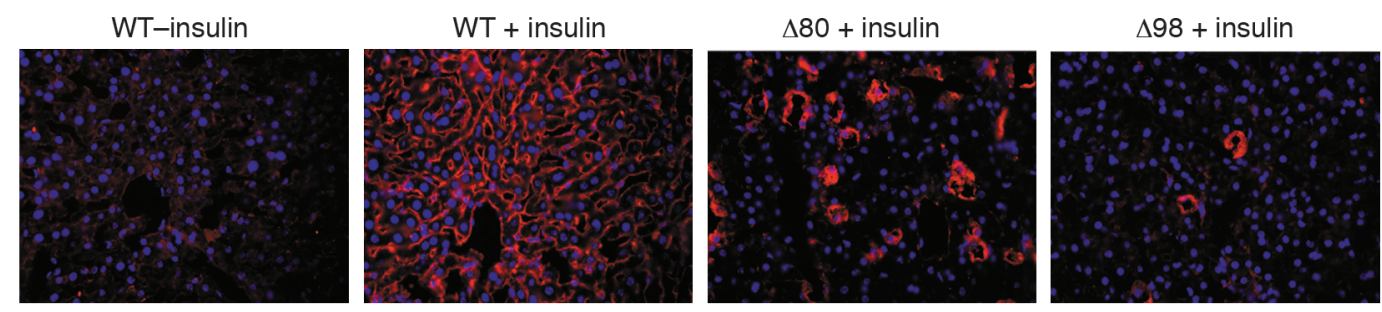

\section{Figure 2}

In vivo generation of PIP3 in response to insulin. We injected insulin via the inferior vena cava of anesthetized mice and after 3 minutes perfused the liver with paraformaldehyde. We then processed the livers for histochemistry with anti-PIP3 antiserum as described. We applied nuclear counterstain to count cell number. We analyzed multiple sections from three mice for each genotype. A representative experiment is shown.

showing the most extensive depletion, muscle tissue and BAT showing intermediate levels, and liver tissue showing the least extensive Insr depletion (Figure 1c). We confirmed the extent of Insr ablation by ${ }^{125}$ I-insulin binding to extracts from various tissues (Table 1). These findings are consistent with previously reported tissue patterns of $\mathrm{Hs}$-Cre-mediated recombination at several additional floxed loci (10).

Demonstration of mosaicism. To assess the extent of mosaicism in vivo, we injected insulin via the portal vein and visualized the generation of PIP3 in the liver by a histochemical method. In WT mice, insulin induced generation of PIP3 and its juxta-membrane localization. In $\Delta 80$ mice, the number of $\mathrm{PIP}^{+}$cells was about $20 \%$; in $\Delta 98$ mice the number was about $2 \%$ of the total (Figure 2). Thus, the degree of genetic mosaicism correlates with the decrease in Insr protein levels and with a decrease in the number of cells in which insulin signaling leads to PIP3 generation. These data indicate that tissues of mosaic mice consist of an admixture of WT and null cells, with few if any cells carrying the Insr heterozygous deletion (see Methods). The data are also consistent with the lack of cellautonomous actions of insulin, at least within the experimental limits of this approach, since there does not appear to be activation of insulin signaling in a greater percentage of cells than expected based on the levels of DNA mosaicism.

Growth retardation and lipoatrophy are common features of $\Delta 80$ and $\Delta 98$ mice. Offspring from both subsets had a normal appearance and weight at birth. However, they failed to thrive thereafter, and attained approximately $30 \%$ of normal weight at 3 weeks (Figure 3 , a and b). The majority of animals died at weaning (about 3 weeks after birth), when insulin requirements increase (19). While none of the $\Delta 80$ mice survived longer than 3 weeks, about $5 \%$ of $\Delta 98$ mice survived as long as 3 months. However, their growth was persistently stunted, and they reached about $20 \%$ of normal weight at that age (Figure $3 \mathrm{c}$ ). Circulating GH levels in 14-dayold mice were normal, whereas IGF1 levels showed a twofold increase in both subsets $(P<0.05$ by ANOVA) (Table 2). We detected a greater than 60 -fold increase in Igfbp1 mRNA levels by real-time-PCR and Northern blotting (Figure 3, $\mathrm{d}$ and e). Serum ligand blotting with ${ }^{125}$ I-IGF2 confirmed the increase in Igfbp1, and showed normal levels of Igfbp2 (Figure 3f) and Igfbp3 (not shown). The extent of growth retardation in $\Delta 98$ survivors is similar to that observed in mice with combined mutations of Ghr and Igfl, which ablate both GH-dependent and GH-independent IGF functions and achieve about $17 \%$ of normal weight (20). However, unlike IGF1-deficient mice, which display both intrauterine and postnatal growth retardation (21), $\Delta 80$ and $\Delta 98$ mice are born of normal size.

Necroscopic and histologic analyses revealed the complete absence of mature adipocytes in the interscapular (BAT), subcutaneous, and epididymal (white adipose tissue, EWAT) regions of both subsets of mice. To simplify presentation, only data on $\Delta 98$ mice are shown (Figure 4a), but we obtained identical data in $\Delta 80$ mice. To ascertain whether the absence of adipocytes was due to impaired pre-adipocyte differentiation or increased lipolysis, we analyzed both areas by EM. Wild-type EWAT consisted of well-developed adipocytes with a single lipid droplet in the cytoplasm. In contrast, EWAT derived from $\Delta 80$ and $\Delta 98$ mice had a fetal/perinatal appearance, with abundant WAT precursors, without evidence of de-lipidated mature adipocytes (22). A peculiar EM finding was the presence of enlarged mitochondria with large electron-dense inclusions (Figure 4, b-d). The nature of these formations is unclear, but they may be related to development of the chondriome (22). Similarly, brown adipocytes in mosaic mice appeared similar to fetal BAT pre-adipocytes (Figure 4, e and f). Although the distinction between pre-adipocytes and de-lipidated mature adipocytes is less well established in BAT than in EWAT, the presence of immature mitochondria with unorganized cristae (Figure 4f) and the lack of tannic acid-reactive material (not shown) are consistent with

\section{Table 1}

125I-insulin binding

\begin{tabular}{lcc}
\hline Genotype & $\Delta 80$ & $\Delta 98$ \\
Liver & $24.7 \pm 1.9$ & $2.9 \pm 0.9$ \\
Brain & $8.1 \pm 0.9$ & $1.9 \pm 0.6$ \\
Muscle & $19.7 \pm 1.8$ & $2.5 \pm 1.1$ \\
Brown adipocytes & $15.6 \pm 1.3$ & $2.1 \pm 0.3$ \\
\hline
\end{tabular}

Quantitation of Insr levels by insulin binding. Numbers represent mean \pm SEM of at least 12 mice for each genotype, expressed as the percentage of binding to extracts from WT mice. 
a
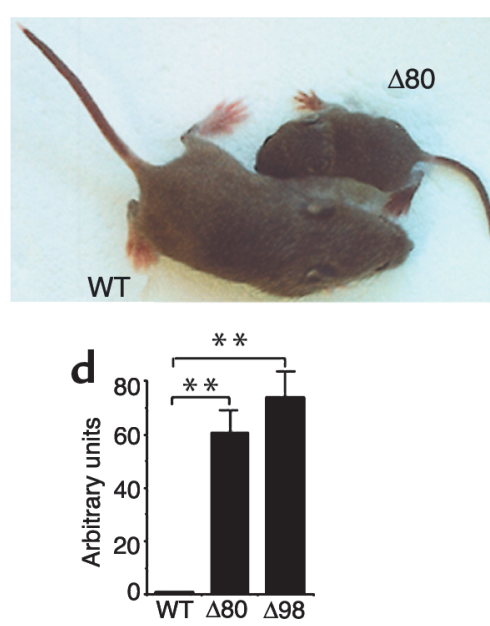

b

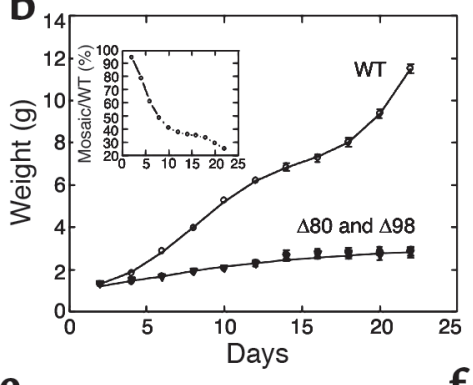

e

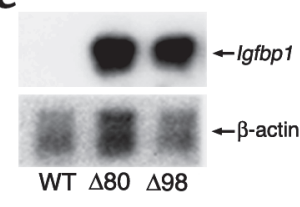

C

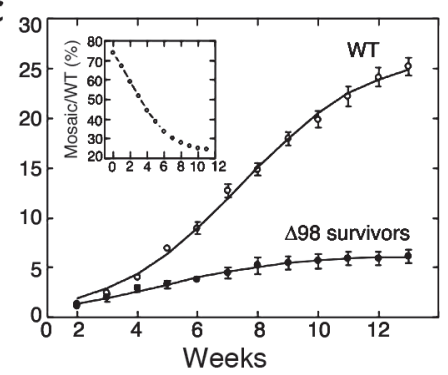

f

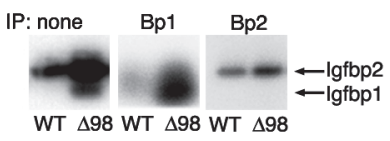

Figure 3

Growth retardation in Insr mosaics. (a) Appearance of 3-week-old mice. (b) Average growth curves of WT and mosaic mice. The curves of the two subsets of mosaics are superimposable. The analysis was terminated at the time of death of $\Delta 80$ mosaics $(n=19$ for WT, 18 for $\Delta 80$, and 15 for $\Delta 98$ ). (c) $\Delta 98$ survivors were followed for up to 13 weeks. The data represent the mean \pm SEM ( $n=28$ for WT and 19 for $\Delta 98$ survivors). The insets present weight ratios of mosaic mice to WT control per time point examined. The decline of these ratios is indicative of lower than normal growth rate for the mosaics throughout the period of observation. (d) Real-time RT-PCR analysis of Igfbp1 expression in liver. We isolated total RNA from WT $(n=20), \Delta 80(n=17)$, and $\Delta 98(n=20)$ mice and subjected it to RT-PCR using a Light Cycler instrument (Roche Perkin-Elmer). We normalized mRNA values using $\beta$-actin as a control. ${ }^{*} P<0.01$. (e) Northern blot analysis of $/ g f b p 1$ expression. We pooled mRNA samples from four different mice for each set and analyzed them by hybridization with an lgfbp 1 probe (upper panel), followed by a $\beta$-actin probe as gel loading control. (f) ${ }^{125}$ I-IGF2 ligand blotting. We obtained serum $(5 \mu$ for each animal) from WT $(n=10)$ and $\Delta 98$ mice $(n=10)$. In the panel on the left, we used whole serum to determine the presence of IGF-binding proteins. As shown, in the middle and right panels, we subjected serum to immunoprecipitation (IP) with the indicated antibodies (Bp1, Bp2) prior to gel electrophoresis and transfer to nylon membranes. We show the region of the gel in the $20-$ to $40-\mathrm{kDa}$ region.

the notion that these are undifferentiated pre-adipocytes (22). In addition, we analyzed expression of key genes in BAT function, including Adrb3, Ucp1, Ppar 7 and its coactivator $P g c 1 \alpha$. Ucp 1 expression decreased by about $95 \%$, $P$ par $\gamma$ by $80 \%$, and $P g c 1 \alpha$ by $60 \%$ in both subsets (Figure $4 \mathrm{~g})$. Since insulin regulates Pgc1 $\alpha$ by both transcriptional and translational mechanisms (23), we also examined Pgc1 $\alpha$ protein levels. Immunoblotting confirmed the decrease in Pgc1 $\alpha$ levels (Figure 4g, lower panels). mRNA levels of Adrb3, a known inducer of Ucp1 expression, were normal in both lines (Figure 4g). These data indicate that lipoatrophy is due to impaired adipocyte differentiation, rather than increased lipolysis.

Hypoglycemia versus hyperglycemia in $\Delta 80$ and $\Delta 98$ mice. We examined several metabolic parameters (Table 2). $\Delta 80$ mice exhibited hypoglycemia associated with an approximately threefold increase in insulin levels. Although the cause of death for both groups of mice is unclear, hypoglycemia is a probable contributor in $\triangle 80$ mice. FFA levels were normal, whereas TG levels were reduced by about 50\%. Hepatic glycogen levels were severely reduced, while muscle glycogen levels were normal. Since mobilization of hepatic glycogen is required to maintain euglycemia during fasting, it is likely that hypoglycemia in $\Delta 80$ mice results from reduced hepatic glycogen content. The hypoglycemia observed in this subset of mice is reminiscent of that seen in the human syndrome of leprechaunism, caused by INSR mutations (24).
In contrast to $\Delta 80$ mice, $\Delta 98$ mice exhibited hyperglycemia and an approximately 13 -fold increase in insulin levels. This metabolic condition is similar to that observed in Insr $\mathrm{KO}$ mice (3). As in $\Delta 80$ mice, hepatic glycogen content was severely decreased. Unlike $\Delta 80$ mice, $\Delta 98$ mice also showed a greater than $80 \%$ decrease in muscle glycogen content (Table 2).

The difference in glucose levels between $\Delta 80$ and $\Delta 98$ mice is noteworthy and could potentially be explained by altered expression of genes involved in hepatic glucose handling. For example, it might have been predicted that hypoglycemic $\Delta 80$ mice had reduced levels of glucogenic enzymes, while hyperglycemic $\Delta 98$ had high levels. Real-time RT-PCR analysis showed that this is not the case (Figure 5a). In fact, Pck1 increased three- to fivefold and G6pc was unchanged in both subsets. The only difference was in Pfkl levels, which were significantly elevated only in $\Delta 98$ mice but are by themselves unlikely to explain the phenotype. Gys1 and Pygl decreased in both $\Delta 80$ and $\Delta 98$ mice. Since these enzymes are important for glycogen synthesis and release, the combined decrease in synthase and phosphorylase cannot explain the decrease in glycogen content. Glut2 (Slc2a2) showed an approximately $40 \%$ decrease exclusively in $\Delta 80$ mice, while Gck levels were unchanged. However, given that even a complete ablation of Glut 2 does not affect hepatic glucose output (25), the observed decrease in $\Delta 80$ mice is unlikely to represent the sole cause of the difference in glucose levels between the two subsets. 
Table 2

Metabolic parameters in Insr mosaics

\begin{tabular}{lcccc}
\hline Genotype & $n$ & WT & $\Delta 80$ & $\Delta 98$ \\
Glucose $(\mathrm{mg} / \mathrm{dl})$ & 40 & $102 \pm 8$ & $47 \pm 6$ & $258 \pm 16$ \\
Insulin $(\mathrm{ng} / \mathrm{ml})$ & 40 & $0.30 \pm 0.04$ & $0.92 \pm 0.21$ & $4.0 \pm 0.9$ \\
$\beta$-cell mass $(\mu \mathrm{g} /$ pancreas $)$ & 3 & $510 \pm 28$ & $128 \pm 17$ & $144 \pm 25$ \\
IGF1 $(\mathrm{ng} / \mathrm{ml})$ & 40 & $430 \pm 49.7$ & $819 \pm 52.3$ & $891 \pm 69.2$ \\
GH $(\mathrm{ng} / \mathrm{ml})$ & 40 & $1.38 \pm 0.33$ & $0.98 \pm 0.22$ & $1.25 \pm 0.30$ \\
FFA $(\mathrm{mEq} / \mathrm{l})$ & 20 & $0.81 \pm 0.13$ & $27 \pm 5$ & $0.78 \pm 0.11$ \\
TG $(\mathrm{mg} / \mathrm{dl})$ & 20 & $56 \pm 2$ & $2.7 \pm 1.0$ & $23 \pm 4$ \\
Hepatic glycogen $(\mathrm{mg} / \mathrm{g})$ & 20 & $14.2 \pm 2.7$ & $1.26 \pm 0.21$ & $0.08 \pm 0.01$ \\
Muscle glycogen $(\mathrm{mg} / \mathrm{g})$ & 20 & $1.42 \pm 0.04$ & $0.26 \pm 0.05$ \\
\hline
\end{tabular}

Overexpression of Foxa2 in the liver of transgenic mice results in postnatal growth retardation, elevated Igfbp1, and reduced hepatic glycogen content (26). To address the possibility that the observed phenotype could be due to altered Foxa2 expression, we measured its mRNA levels. In both subsets of mice, Foxa2 was decreased, thus ruling out its involvement in this phenotype. On the other hand, the forkhead transcription factor Foxo1 promotes Pck1, G6pc, and Igfbp1 expression, and is inhibited by insulin via Akt-mediated phosphorylation (14). Foxo1 mRNA (not shown) and protein levels were normal in both subsets, but the phosphate content of the key Akt phosphorylation site was decreased by about $40 \%$ and $60 \%$ in $\Delta 80$ and $\Delta 98$ mice, respectively, consistent with increased transcriptional activity (Figure $5 \mathrm{~b}$ ). Thus, the increase in glycogen mobilization can be explained in part by unrestrained Foxo1 activity. Among the Foxo 1 targets, the transcriptional co-activator $P g c 1 \alpha$ (27) was increased by twofold and fourfold in $\Delta 80$ and $\Delta 98$ mice, respectively (Figure $5 \mathrm{a}$ ). Pgc1 $\alpha$ cooperates with Foxo1 to increase Pck1 and G6pc transcription (7), and the observed increase is likely to represent a compensatory response to increase gluconeogenesis, as observed with prolonged fasting (28). To summarize these data, both $\Delta 80$ and $\Delta 98$ mice had similar alterations of hepatic gene expression and glycogen content. Thus, the difference in circulating glucose levels cannot be explained by liver metabolism.

We next analyzed insulin signaling in muscle extracts from the two sets of mosaics. In $\Delta 80$ mice, insulin-depend- ent co-precipitation of the p85 subunit of PI3K with antiphosphotyrosine antiserum was reduced by about $80 \%$, as was the amount of phospho-Akt. In $\Delta 98$ mice, there was a greater than $90 \%$ decrease in $\mathrm{p} 85$ co-precipitation, while no phospho-Akt could be detected in response to insulin (Figure 6). Thus, muscle insulin signaling is decreased in proportion to the degree of Insr mosaicism.

The small size of these mice preempts further metabolic analyses. Nevertheless, based on these observations, we suggest the following model for the difference in glucose levels between $\Delta 80$ and $\Delta 98$ mice. In the liver, both groups of mice are equally insulin-resistant, as demonstrated by the comparable expression of metabolic control genes and glycogen levels. Hepatic insulin resistance causes inability to synthesize and store glycogen; this explains the fasting hypoglycemia in $\Delta 80$ mice. In contrast to liver, biochemical data indicate that residual insulin signaling is present in $\Delta 80$ mice but not in $\Delta 98$ mice, as demonstrated by phospho-Akt levels. Consistent with this observation, muscle glycogen content is normal in $\Delta 80$ mice but reduced in $\Delta 98$ mice. These data indicate that $\Delta 80$ mice are insulin-sensitive in the muscle, whereas $\Delta 98$ are insulin-resistant. Sensitivity to insulin in the muscle would be expected to exacerbate hypoglycemia in $\Delta 80$ mice, because glucose is cleared from the blood and taken up by skeletal muscle. In contrast, resistance to insulin action in the muscle of $\Delta 98$ mice results in impaired glucose uptake and hyperglycemia. In summary, we propose that the difference between the two strains is due to tissuespecific differences in insulin resistance. While in theory

Table 3

Phenotypic comparison between Insr mosaics and leprechaunism

\begin{tabular}{|c|c|c|c|c|}
\hline & Leprechaunism & $\Delta 80$ & $\Delta 98$ & Insr KO \\
\hline Growth & Prenatal and postnatal retardation & Postnatal retardation & Postnatal retardation & Near normal (90\%) \\
\hline Life span & $<1$ year & 3 weeks & 3 weeks (95\%), 3 months ( $5 \%)$ & $<1$ week \\
\hline Glucose & Low & Low & High & High \\
\hline Insulin & $\begin{array}{c}\text { High } \\
\text { (up to } 1,000 \text {-fold) }\end{array}$ & $\begin{array}{c}\text { High } \\
\text { (one- to threefold) }\end{array}$ & $\begin{array}{c}\text { High } \\
\text { (2- to } 20 \text {-fold) }\end{array}$ & $\begin{array}{c}\text { High } \\
\text { (5- to } 50 \text {-fold) }\end{array}$ \\
\hline Ketoacidosis & Transient & Absent & Constant & Constant \\
\hline Hepatic steatosis & Moderate & Moderate & Severe & Severe \\
\hline Lipoatrophy & Present & Present & Present & Present \\
\hline
\end{tabular}



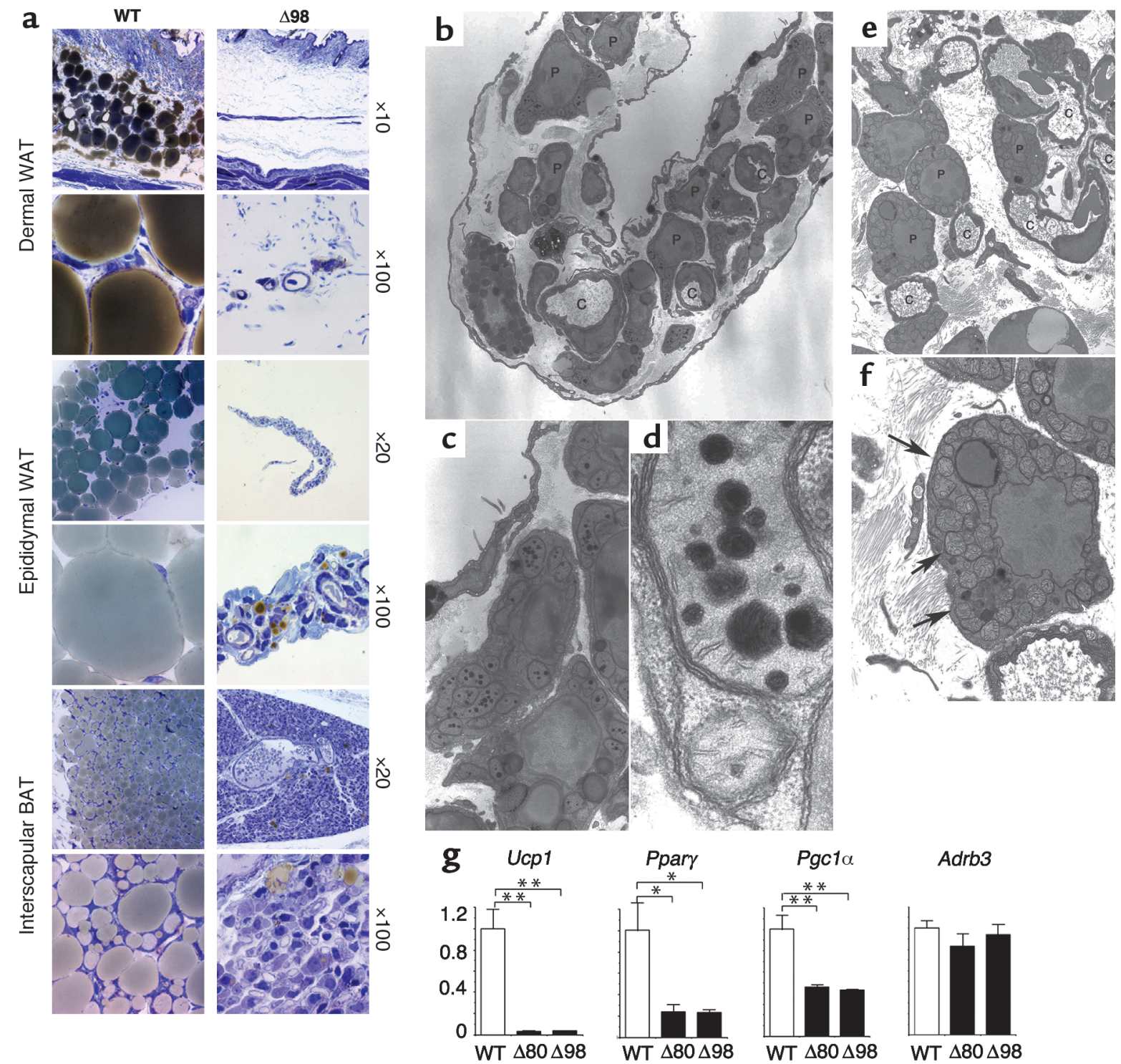

Adrb3

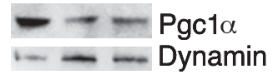

\section{Figure 4}

Lipoatrophy and analysis of BAT gene expression. (a) Representative histologic appearance of H\&E-stained sections from dermal WAT, periepididymal WAT, and BAT. For simplicity, only $\Delta 98$ mice are shown, but the data are identical in $\Delta 80$ mice. (b-d) EM analysis of peri-epididymal WAT precursors in $\Delta 98$ mice. The magnifications shown are $(\mathbf{b}) \times 1,900,(\mathbf{c}) \times 5,000$, and $(\mathbf{d}) \times 46,000$. Intramitochondrial inclusions of electron-dense material are shown in $\mathbf{b}$ and $\mathbf{d}$. The mitochondria are enlarged and show poorly organized cristae. (e and $\mathbf{f}$ ) EM analysis of BAT precursors in $\Delta 98$ mice. Numerous pre-adipocytes adjacent to small capillaries can be seen. Mitochondria are indicated by arrows. The magnifications shown are $(\mathbf{e}) \times 1,900$ and $(\mathbf{f}) \times 5,000$. P, pre-adipocytes; C, capillaries; L, lipid droplet. $(\mathbf{g})$ Analysis of BAT gene expression. We isolated mRNA from 3-week-old mice and performed real-time RT-PCR with primers encoding the genes indicated at the top of each panel. The data represent means \pm SEM of three independent measurements $(n=10$ for each genotype). We used amplification of $\beta$-actin to normalize gene expression data. Asterisks indicate a statistically significant difference ( ${ }^{*} P<0.05$ and $* * P<0.01$ by ANOVA) between genotypes. To measure the levels of immunoreactive Pgc1 $\alpha$, we performed Western blotting with anti-Pgc1 $\alpha$ antiserum. We show a representative autoradiogram and a loading control with anti-dynamin antiserum below the Pgc1 $\alpha$ real-time PCR graph.

the difference could be due to variations in the counterregulatory response between the two sets of mice, the presence of normal GH levels and the normal glucagon histochemistry (Figure 7) make that interpretation less likely.

Lipoatrophy without dyslipidemia in $\Delta 80$ and $\Delta 98$ mice. Lipoatrophy is generally associated with severe hyperin- sulinemia, hepatic steatosis, and increased FFA and TG levels $(29,30)$. However, fatty liver infiltration was absent in $\Delta 80$ mice and modest in $\Delta 98$ mice (Figure $5 \mathrm{c}$ ). Moreover, FFA and TG levels were normal or low in both subsets, and insulin levels were only moderately elevated in $\Delta 98$ mice (Table 2). To determine the mechanism of this 
dissociation between lipoatrophy and lipid abnormalities, we measured expression of the transcription factor Srebf1, which regulates expression of lipogenic (31) and gluconeogenetic (32) genes in an insulin-dependent manner (33). In both subsets, we found a substantial decrease in Srebf1 expression (Figure 5a), suggesting that the lack of hyperlipidemia in Insr mosaics is due to reduced Srebf1-mediated lipid synthesis.

Lack of compensatory $\beta$-cell hyperplasia in $\Delta 98$ mice. We next examined pancreatic histomorphometry in both lines. We could not recover islets from $\Delta 80$ or $\Delta 98$ mice to measure the degree of Insr mosaicism, because of their small size. Nevertheless, given the common endodermal derivation of liver and pancreas (34), it is fair to assume that the degree of cellular mosaicism in pancreatic islets mirrored that seen in liver tissue. Despite the mild-to-moderate increases in insulin levels, islet size and morphology in mosaic animals were similar to those in WT mice (Figure 7), and $\beta$-cell mass was proportional to pancreas size (Table 1). Thus, given the hyperglycemia in $\Delta 98$ mice, $\beta$-cell mass should be considered inappropriately small. Indeed, the number of $\beta$ cells with nuclear expression of the insulin gene transcription factor Pdx1 decreased in approximate proportion to the degree of Insr mosaicism. Interestingly, in islets of $\Delta 98$ mice, Pdx1 often mislocalized to the cytoplasm, but the significance of this observation is controversial (35). We recently reported that Foxo1 regulates $P d x 1$ expression in pancreatic $\beta$ cells (36). Whereas Foxo1 showed variable subcellular distribution in WT $\beta$ cells, it showed exclusive nuclear localization in $\beta$ cells from $\Delta 98$ mice (Figure 7). These data can be interpreted to suggest that the probable impairment of insulin signaling in $\beta$ cells prevents $\beta$-cell compensation to insulin resistance, without affecting fetal $\beta$-cell development and their postnatal secretory function.

\section{Discussion}

We draw two main conclusions from our mosaic analysis of Insr function. First, different biological responses to insulin appear to have different sensitivities to reduction of Insr signaling. Thus, it appears that insulin-dependent growth, adipogenesis, and hepatic glycogen synthesis are more sensitive to depletion of Insr than muscle glycogen synthesis, which in turn probably reflects decreased insulin-dependent glucose uptake. Second, despite the profound depletion of Insr, mosaic mice developed few if any of the metabolic consequences of insulin resistance, such as $\beta$-cell hyperplasia, obesity, and dyslipidemia.

Studies of insulin-induced PIP3 generation in mosaic mice show a close correlation between the number of a
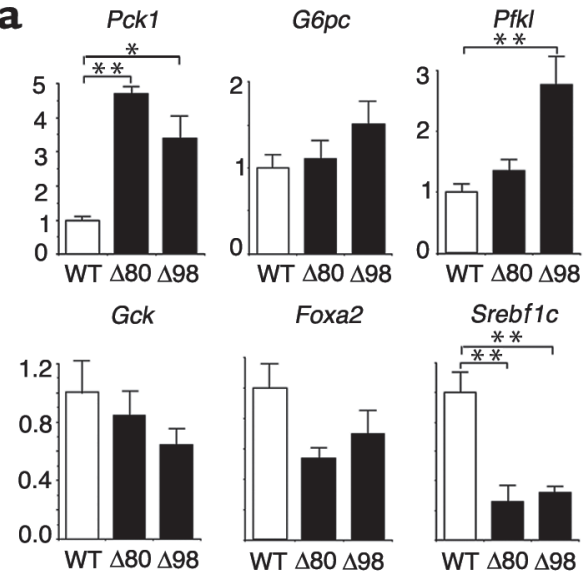

c

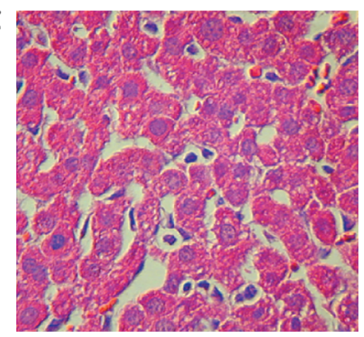

WT

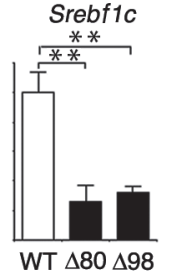

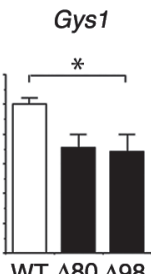

b

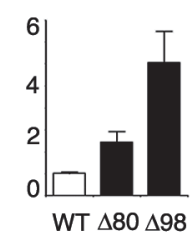

Slc2a2
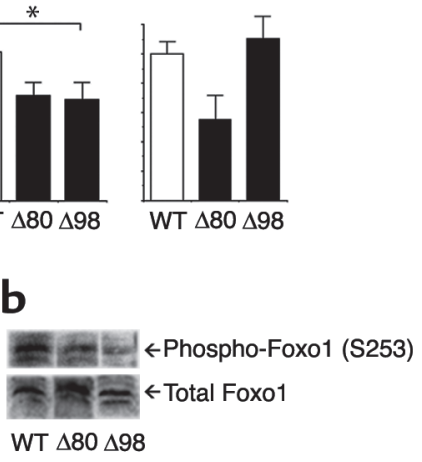

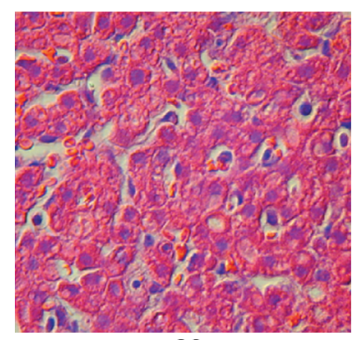

$\Delta 80$

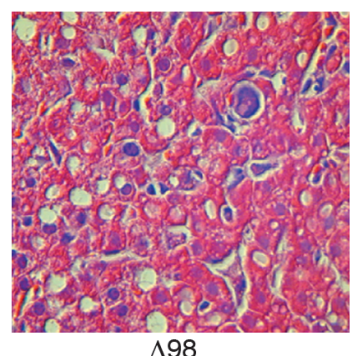

$\triangle 98$

\section{Figure 5}

Analysis of liver gene expression, Foxo1 phosphorylation, and histology. (a) Real-time RT-PCR. We isolated mRNA from the liver of 3-weekold mice and performed real-time RT-PCR with primers encoding the genes indicated at the top of each bar graph. The data represent means \pm SEM of three independent measurements $\left(n=14\right.$ for each genotype). ${ }^{*} P<0.05$. ${ }^{*} P<0.01$. (b) Foxo 1 phosphorylation. We removed the livers from WT, $\Delta 80$, and $\Delta 98$ mice and isolated proteins by detergent extraction. We performed Western blotting with anti-phospho $\mathrm{S} 253$ Foxo1 antibody (upper panel), and then probed the filter again with anti-Foxo1 antibody (lower panel). We show a representative experiment. (c) H\&E-stained liver sections from WT, $\Delta 80$, and $\Delta 98$ mice. 


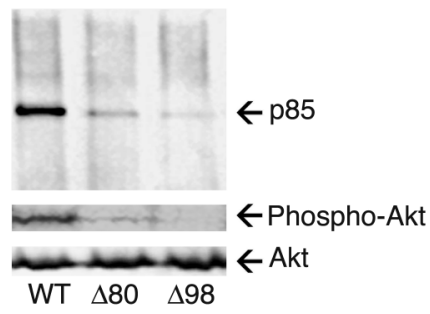

Figure 6

Muscle insulin signaling. Mice were treated with insulin, delivered via the inferior vena cava, and protein extracts were prepared and analyzed by immunoprecipitation with anti-phosphotyrosine antiserum followed by immunoblotting with anti-p85 antiserum (upper panel). Alternatively, extracts were resolved on SDS-PAGE and analyzed by immunoblotting with anti-pSer ${ }^{473}$-Akt antiserum (middle panel), followed by reprobing of the stripped blots with anti-Akt antiserum (bottom panel).

$\mathrm{PIP}^{+}$cells and the degree of mosaicism. While technical limitations prevent us from demonstrating that the $\mathrm{PIP}^{+}$cells are also Insr ${ }^{+}$, this appears to be extremely likely. These data support two conclusions: first, that the tissues of $\Delta 80$ and $\Delta 98$ mice consist of an admixture of WT and Insr null cells, with few if any cells carrying a heterozygous Insr deletion. Second, the findings are consistent with a cell-autonomous mechanism of Insr action, in which the phenotype is directly determined by Insr ablation in the target cell, without compensatory mechanisms by which normal cells can send signals to cells lacking Insr.

Growth retardation. At about $30 \%$ of normal weight, $\Delta 80$ and $\Delta 98$ mice are the most severely growthretarded mice generated by ablating insulin signaling (37). Unlike embryos lacking both IGF1 and
IGF2 signaling, which achieve $30 \%$ of normal weight (21), the birthweight of $\Delta 80$ and $\Delta 98$ mice is nearly normal. This observation is consistent with the late onset of the growth-promoting effects of Insr during mouse gestation (4). The likeliest mechanism of the postnatal growth retardation is through increased hepatic Igfbp1, which is known to bind circulating IGF1 and limit its bioavailability (38). Indeed, Igfbp1 overexpression in transgenic mice can cause growth retardation (39), although there are no precedents for such a large increase in Igfbp1 levels as seen in Insr mosaics. For example, liver-specific ablation of Insr is not associated with a substantial increase of Igfbp1 levels (40), possibly because, in those experiments, ablation of Insr occurs gradually over the first few weeks of postnatal life (41). Indeed, there is evidence in both humans (42) and rats (43) that Igfbp1 levels are negatively correlated with "free" IGF1, the main determinant of linear growth (44). Our data provide evidence that insulin regulates growth independently of metabolism, and indirectly support the notion that free circulating IGF1 contributes to growth (45).

Lipoatrophy. The lack of WAT could be due either to a failure to differentiate adipocytes or to unrestrained lipolysis, leading to TG depletion. Although the distinction between the two entities is at times difficult and arbitrary, our EM data indicate that mosaic mice possess pre-adipocytes but not mature adipocytes. The failure of most pre-adipocytes to differentiate is consistent with the demonstrated role of Insr in this process (46-48). This phenotype is entirely different from that caused by conditional inactivation of Insr in mature adipocytes through the aP2 promoter (49). In that case, adipocytes undergo differentiation but dis-
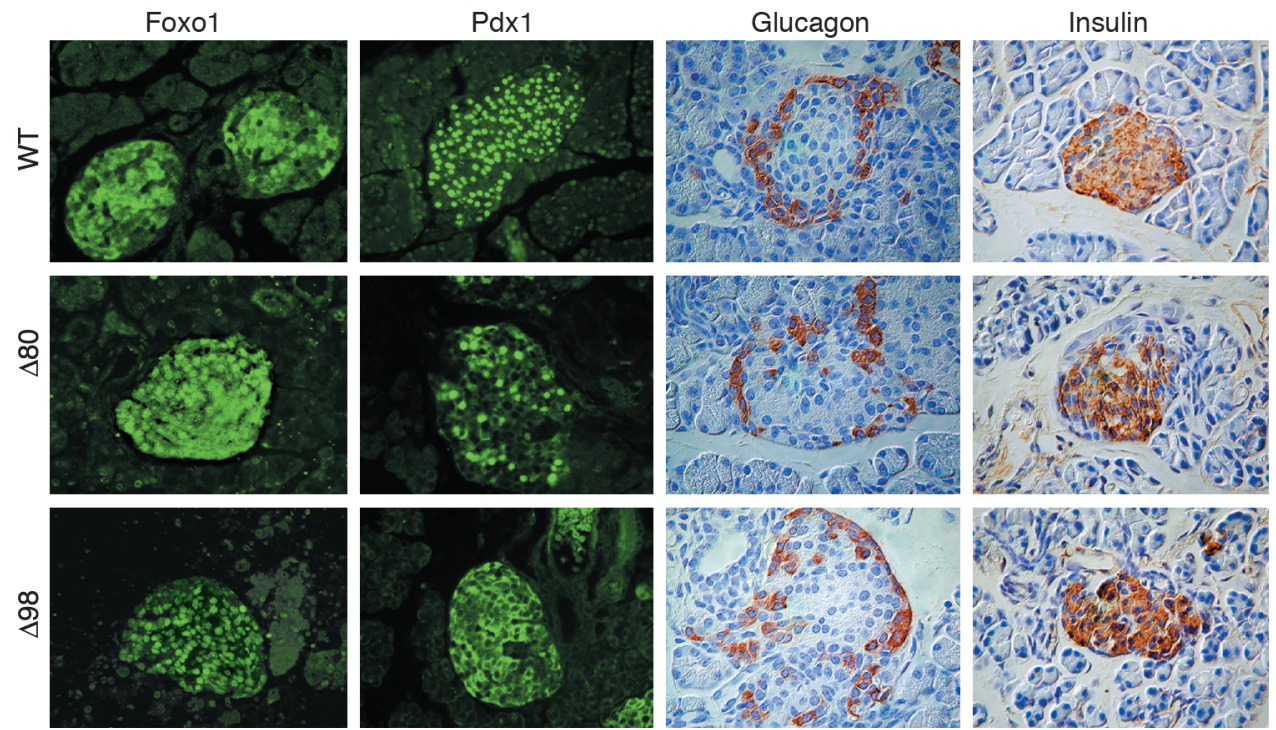

\section{Figure 7}

Immunohistochemistry of pancreatic islets. We isolated pancreata from 3-week-old mice and processed them for immunohistochemistry with anti-insulin and anti-glucagon antisera. We used frozen sections for anti-Pdx1 and anti-Foxo1 immunostaining. Note the different intracellular distribution of $\mathrm{Pdx} 1$ and Foxo 1 between WT and $\Delta 98$ mice, and the intermediate pattern in $\Delta 80$ mice. 
play size heterogeneity. These data, like those mentioned above in mice with liver-specific Insr knockout, underscore the importance of distinguishing between conditional knockouts introduced at different developmental stages in the same cell type (8).

Lipoatrophy is generally associated with profound insulin resistance. However, unlike prior models of lipoatrophy $(29,30)$, but similar to the double mutants lacking Irs1 and Irs3 (50), Insr mosaics lack $(\Delta 80$ mice) or display modest insulin resistance $(\Delta 98$ mice). This dissociation indicates that the development of insulin resistance in lipoatrophy results from the combination of impaired fat storage in adipose tissue and preserved insulin sensitivity in other tissues, primarily the the liver (31). The failure to form an adequate fat mass in Insr mosaics appears to be due to reduced expression of key pro-adipogenic genes, since we detected very low levels of Ucp1, Ppar $\gamma$, and $P g c 1 \alpha$ in BAT. This observation is consistent with the role of Insr in adipogenesis (46-48).

$\beta$-cell compensation to insulin resistance. Insulin resistance is associated with hyperinsulinemia and leads to $\beta$-cell hyperplasia. The lack of compensatory $\beta$-cell hyperplasia in Insr mosaics could be construed to suggest that insulin signaling is required for this response. These data should be viewed in the context of emerging evidence indicating that insulin signaling regulates various aspects of $\beta$-cell function, including proliferation and hormone secretion (51). We have previously shown that the transcription factor Foxo 1 is a negative regulator of $\beta$-cell proliferation (14). Intriguingly, we now report increased nuclear localization of Foxo1 in $\beta$ cells of $\Delta 98$ mice. These findings are consistent with the view that the lack of $\beta$-cell hyperplasia in this model is due to impaired insulin signaling in $\beta$ cells and/or their progenitors. Although it is possible that the decrease is due to impaired Igf1 signaling, the recent demonstration that ablation of the Igf1 receptor does not affect $\beta$-cell proliferation makes this explanation less likely $(17,52)$. It should be emphasized, however, that the mouse and human models differ in this respect, since children with leprechaunism are more profoundly hyperinsulinemic. Therefore, it remains to be determined whether a similar function can be ascribed to Insr signaling in the human pancreas (6). Leprechaunism explained? In humans, mutations ablating Insr function give rise to leprechaunism, an invariably fatal syndrome of extreme insulin resistance with growth retardation, lipoatrophy, and hypoglycemia (6). It is unclear why patients with leprechaunism develop hypoglycemia (24). $\Delta 80$ mice represent a faithful model of this human syndrome (Table 3). Based on the proposed explanation for the hypoglycemia in $\Delta 80$ mice, we suggest that a similar mechanism of impaired insulin action in liver and fat tissue, with preserved insulin sensitivity in muscle tissue, underlies the pathogenesis of hypoglycemia in children with leprechaunism.

Finally, we suggest that future treatment strategies for insulin resistance should strike a balance between improving insulin sensitivity and preventing excessive insulin action by selective targeting of the biologic responses to insulin in different tissues.

\section{Acknowledgments}

This work was supported by NIH DK58282 grant and Juvenile Diabetes Research Foundation grant 200-893. T.K. is the recipient of a Juvenile Diabetes Foundation Postdoctoral Fellowship. We thank Youping Liu for skilled technical assistance with immunohistochemistry and members of the Accili laboratory for critical discussion of the data.

1. Saltiel, A.R., and Kahn, C.R. 2001. Insulin signalling and the regulation of glucose and lipid metabolism. Nature. 414:799-806.

2. Kim, J., and Accili, D. 2002. Signalling through IGF-I and insulin receptors: where is the specificity? Growth Horm. IGF Res. 12:84-90.

3. Accili, D., et al. 1996. Early neonatal death in mice homozygous for a null allele of the insulin receptor gene. Nat. Genet. 12:106-109.

4. Louvi, A., Accili, D., and Efstratiadis, A. 1997. Growth-promoting interaction of IGF-II with the insulin receptor during mouse embryonic development. Dev. Biol. 189:33-48.

5. Liu, J.P., Baker, J., Perkins, A.S., Robertson, E.J., and Efstratiadis, A. 1993. Mice carrying null mutations of the genes encoding insulin-like growth factor I (Igf-1) and type 1 IGF receptor (Igf1r). Cell. 75:59-72.

6. Nakae, J., Kido, Y., and Accili, D. 2001. Distinct and overlapping functions of insulin and IGF-I receptors. Endocr. Rev. 22:818-835.

7. Puigserver, P., et al. 2003. Insulin-regulated hepatic gluconeogenesis through FOXO1-PGC-1alpha interaction. Nature. 423:550-555.

8. Kitamura, T., Kahn, C.R., and Accili, D. 2003. Insulin receptor knockout mice. Ann. Rev. Physiol. 65:313-332.

9. Kim, J.K., et al. 2000. Redistribution of substrates to adipose tissue promotes obesity in mice with selective insulin resistance in muscle. J. Clin. Invest. 105:1791-1797.

10. Dietrich, P., Dragatsis, I., Xuan, S., Zeitlin, S., and Efstratiadis, A. 2000. Conditional mutagenesis in mice with heat shock promoter-driven Cre transgenes. Mamm. Genome. 11:196-205.

11. Bruning, J.C., et al. 1998. A muscle-specific insulin receptor knockout exhibits features of the metabolic syndrome of NIDDM without altering glucose tolerance. Mol. Cell. 2:559-569.

12. Kitamura, T., et al. 2001. Preserved pancreatic beta-cell development and function in mice lacking the insulin receptor-related receptor. Mol. Cell. Biol. 21:5624-5630.

13. Chiao, E., et al. 2002. Overgrowth of a mouse model of the SimpsonGolabi-Behmel syndrome is independent of IGF signaling. Dev. Biol. 243:185-206.

14. Nakae, J., et al. 2002. Regulation of insulin action and pancreatic betacell function by mutated alleles of the gene encoding forkhead transcription factor Foxo1. Nat. Genet. 32:245-253.

15. Accili, D., et al. 1989. A mutation in the insulin receptor gene that impairs transport of the receptor to the plasma membrane and causes insulin-resistant diabetes. EMBO J. 8:2509-2517.

16. Ooi, G.T., Tseng, L.Y., Tran, M.Q., and Rechler, M.M. 1992. Insulin rapidly decreases insulin-like growth factor-binding protein-1 gene transcription in streptozotocin-diabetic rats. Mol. Endocrinol. 6:2219-2228.

17. Xuan, S., et al. 2002. Defective insulin secretion in pancreatic $\beta$ cells lacking type 1 IGF receptor. J. Clin. Invest. 110:1011-1019. doi:10.1172/JCI200215267.

18. Sauer, B. 1998. Inducible gene targeting in mice using the Cre/lox system. Methods. 14:381-392.

19. Girard, J., Ferre, P., Pegorier, J.P., and Duee, P.H. 1992. Adaptations of glucose and fatty acid metabolism during perinatal period and sucklingweaning transition. Physiol. Rev. 72:507-562.

20. Lupu, F., Terwilliger, J.D., Lee, K., Segre, G.V., and Efstratiadis, A. 2001 Roles of growth hormone and insulin-like growth factor 1 in mouse postnatal growth. Dev. Biol. 229:141-162.

21. Baker, J., Liu, J.P., Robertson, E.J., and Efstratiadis, A. 1993. Role of insulin-like growth factors in embryonic and postnatal growth. Cell. 75:73-82.

22. Barnard, T. 1969. The ultrastructural differentiation of brown adipose tissue in the rat. J. Ultrastruct. Res. 29:311-322.

23. Tsukiyama-Kohara, K., et al. 2001. Adipose tissue reduction in mice lacking the translational inhibitor 4E- BP1. Nat. Med. 7:1128-1132.

24. Taylor, S.I. 1992. Lilly Lecture: molecular mechanisms of insulin resistance. Lessons from patients with mutations in the insulin-receptor gene. Diabetes. 41:1473-1490.

25. Guillam, M.T., Burcelin, R., and Thorens, B. 1998. Normal hepatic glucose production in the absence of GLUT2 reveals an alternative path- 
way for glucose release from hepatocytes. Proc. Natl. Acad. Sci. U. S. A. 95:12317-12321.

26. Rausa, F.M., et al. 2000. Elevated levels of hepatocyte nuclear factor 3 beta in mouse hepatocytes influence expression of genes involved in bile acid and glucose homeostasis. Mol. Cell. Biol. 20:8264-8282.

27. Daitoku, H., Yamagata, K., Matsuzaki, H., Hatta, M., and Fukamizu, A. 2003. Regulation of PGC-1 promoter activity by protein kinase $B$ and the forkhead transcription factor FKHR. Diabetes. 52:642-649.

28. Yoon, J.C., et al. 2001. Control of hepatic gluconeogenesis through the transcriptional coactivator PGC-1. Nature. 413:131-138.

29. Moitra, J., et al. 1998. Life without white fat: a transgenic mouse. Genes Dev. 12:3168-3181.

30. Shimomura, I., et al. 1998. Insulin resistance and diabetes mellitus in transgenic mice expressing nuclear SREBP-1c in adipose tissue: model for congenital generalized lipodystrophy. Genes Dev. 12:3182-3194.

31. Shimomura, I., et al. 2000. Decreased IRS-2 and increased SREBP-1c lead to mixed insulin resistance and sensitivity in livers of lipodystrophic and ob/ob mice. Mol. Cell. 6:77-86.

32. Chakravarty, K., et al. 2001. Sterol regulatory element-binding protein$1 \mathrm{c}$ mimics the negative effect of insulin on phosphoenolpyruvate carboxykinase (GTP) gene transcription. J. Biol. Chem. 276:34816-34823.

33. Fleischmann, M., and Iynedjian, P.B. 2000. Regulation of sterol regulatory-element binding protein 1 gene expression in liver: role of insulin and protein kinase B/cAkt. Biochem. J. 349:13-17.

34. Slack, J.M. 1995. Developmental biology of the pancreas. Development. 121:1569-1580.

35. Macfarlane, W.M., et al. 1999. Glucose stimulates translocation of the homeodomain transcription factor PDX1 from the cytoplasm to the nucleus in pancreatic beta-cells. J. Biol. Chem. 274:1011-1016.

36. Kitamura, T., et al. 2002. The forkhead transcription factor Foxo1 links insulin signaling to $P d x 1$ regulation of pancreatic $\beta$ cell growth. J. Clin. Invest. 110:1839-1847. doi:10.1172/JCI200216857.

37. Efstratiadis, A. 1998. Genetics of mouse growth. Int. J. Dev. Biol. 42:955-976

38. Clemmons, D.R. 1998. Role of insulin-like growth factor binding proteins in controlling IGF actions. Mol. Cell. Endocrinol. 140:19-24.

39. Schneider, M.R., Lahm, H., Wu, M., Hoeflich, A., and Wolf, E. 2000.
Transgenic mouse models for studying the functions of insulin-like growth factor-binding proteins. FASEB J. 14:629-640.

40. Michael, M.D., et al. 2000. Loss of insulin signaling in hepatocytes leads to severe insulin resistance and progressive hepatic dysfunction. Mol. Cell. 6:87-97.

41. Postic, C., and Magnuson, M.A. 2000. DNA excision in liver by an albumin-Cre transgene occurs progressively with age. Genesis. 26:149-150.

42. Frystyk, J., Grofte, T., Skjaerbaek, C., and Orskov, H. 1997. The effect of oral glucose on serum free insulin-like growth factor-I and -II in health adults. J. Clin. Endocrinol. Metab. 82:3124-3127.

43. Frystyk, J., et al. 1998. Developmental changes in serum levels of free and total insulin-like growth factor I (IGF-I), IGF-binding protein-1 and -3, and the acid-labile subunit in rats. Endocrinology. 139:4286-4292.

44. Yakar, S., et al. 2002. Circulating levels of IGF-1 directly regulate bone growth and density. J. Clin. Invest. 110:771-781. doi:10.1172/ JCI200215463.

45. D'Ercole, A.J., and Calikoglu, A.S. 2001. Editorial review: the case of local versus endocrine IGF-I actions: the jury is still out. Growth Horm. IGF Res. 11:261-265.

46. Accili, D., and Taylor, S.I. 1991. Targeted inactivation of the insulin receptor gene in mouse 3T3-L1 fibroblasts via homologous recombination. Proc. Natl. Acad. Sci. U. S. A. 88:4708-4712.

47. Entingh, A.J., Taniguchi, C.M., and Kahn, C.R. 2003. Bi-directional regulation of brown fat adipogenesis by the insulin receptor. J. Biol. Chem. 278:33377-33383.

48. Nakae, J., et al. 2003. The forkhead transcription factor Foxo1 regulates adipocyte differentiation. Dev. Cell. 4:119-129.

49. Bluher, M., et al. 2002. Adipose tissue selective insulin receptor knockout protects against obesity and obesity-related glucose intolerance. Dev. Cell. 3:25-38.

50. Laustsen, P.G., et al. 2002. Lipoatrophic diabetes in Irs1(-/-)/Irs3(-/-) double knockout mice. Genes. Dev. 16:3213-3222.

51. Accili, D. 2001. A kinase in the life of the $\beta$ cell. J. Clin. Invest. 108:1575-1576. doi:10.1172/JCI200114454.

52. Kulkarni, R.N., et al. 2002. Beta-cell-specific deletion of the Igf1 receptor leads to hyperinsulinemia and glucose intolerance but does not alter beta-cell mass. Nat. Genet. 31:111-115. 